\title{
Clinical Significance of the Resistive Index of Prostatic Blood Flow According to Prostate Size in Benign Prostatic Hyperplasia
}

\author{
Se Yun Kwon ${ }^{1}$, Jung Woo Ryu ${ }^{1}$, Dai Hai Choi ${ }^{2}$, Kyung Seop Lee ${ }^{1}$ \\ ${ }^{1}$ Department of Urology, Dongguk University College of Medicine, Gyeongju, Korea \\ ${ }^{2}$ Department of Emergency Medicine, Dongguk University College of Medicine, Gyeongju, Korea
}

\begin{abstract}
Purpose: The authors evaluated the relationships between the clinical factors and resistive indexes (RIs) of prostate and urethral blood flows by using power Doppler transrectal ultrasonography (PDUS) in men with benign prostatic hyperplasia (BPH).

Methods: The data of 110 patients with BPH and lower urinary tract symptoms (LUTS) treated between January 2015 and July 2015 were prospectively collected. PDUS was used to identify the capsular and urethral arteries of the prostate in order to measure RIs. International Prostate Symptom Score (IPSS), maximal flow rate (Qmax), total prostate volume (TPV), transition zone volume (TZV), transition zone index (=TZV/TPV), presence of intravesical prostatic protrusion (IPP), and the RIs of capsular and urethral arteries were evaluated for all of the patients by one urologist.

Results: The 110 patients were categorized according to IPSS (mild symptoms, 0-7; moderate symptoms, 8-19; and severe symptoms, 20-35), Qmax ( $<10$ and $\geq 10 \mathrm{~mL} / \mathrm{sec}$ ), TPV ( $<30$ and $\geq 30 \mathrm{~mL}$ ), and presence or absence of IPP. No significant relationship was found between the mean RI of any artery and IPSS or Qmax. The mean RIs of the urethral artery, and left and right capsular arteries were significantly dependent on prostate size and the presence of IPP.

Conclusions: RI obtained by using PDUS correlated with the presence of IPP and prostate size. The RI of prostate blood flow can be used as a noninvasive diagnostic tool for BPH with LUTS.
\end{abstract}

Keywords: Resistive Index; Prostate; Prostate Hyperplasia

- Research Ethics: This study was approved by Institutional Review Board of Dongguk University College of Medicine (110757-201501-HR-02-03).

- Conflict of Interest: No potential conflict of interest relevant to this article was reported.

\section{INTRODUCTION}

Benign prostatic hyperplasia (BPH) is a disease primarily of middle-aged and elderly men [1,2]. Health-care utilization due to the increasing incidence of $\mathrm{BPH}$ caused by societal aging is rapidly increasing in South Korea, especially among the elderly [3-5]. BPH is a progressive disease, and BPH patients often present with lower urinary tract symptoms (LUTS) [6,7], which impair health-related quality of life. Transrectal ultrasonography (TRUS) provides a useful means of assessing BPH patients [8-10]. The Korean Prostate Society recommends TRUS as an optional study for BPH patients. Ultrasonographic technology has made remarkable progress, and recent publications suggest that Doppler ultrasonography is useful for evaluating prostatic disease because it can be used to visualize the prostatic vascular architecture [11]. Neumaier et al. [12] depicted the vascular

Corresponding author: Kyung Seop Lee (iD http://orcid.org/0000-0002-0554-4272 Department of Urology, Dongguk University Gyeongju Hospital, Dongguk University College of Medicine, 87 Dongdae-ro, Gyeongju 38067, Korea E-mail: ksleemd@dongguk.ac.kr / Tel: +82-54-770-8265 / Fax: +82-54-770-8378 Submitted: July 22, 2015 / Accepted after revision: August 25, 2015 
anatomy of the normal prostate by using transrectal color Doppler imaging and explained in detail how urethral and capsular arteries are anatomically distributed in the prostate. Several reports have shown the diagnostic benefit of resistive indexes $(\mathrm{RIs}=$ [peak systolic velocity-end diastolic velocity]/peak systolic velocity) measured by using Doppler imaging [13-15]. However, few reports have described the application of Doppler imaging in $\mathrm{BPH}$ patients.

This study was undertaken to evaluate the relationships between the RIs of the capsular and urethral arteries and BPH parameters.

\section{MATERIALS AND METHODS}

From January 2015 to July 2015, we prospectively collected and analyzed data of patients with BPH who complained of LUTS. Those who had elevated prostate specific antigen (PSA) levels (>4.0 ng/mL), had abnormal digital rectal examination results, and previously received medical BPH/LUTS therapy within 6 months, including $\alpha$-blockers and $5 \alpha$-reductase inhibitors and underwent surgery of the prostate and bladder neck or urethra were excluded. Informed consent was provided by all the study subjects, and the study was approved by Institutional Review Board of Dongguk University College of Medicine (110757201501-HR-02-03).

The 110 study subjects were assessed by history taking and divided according to International Prostate Symptom Score (IPSS; mild symptoms, 0-7; moderate symptoms, 8-19; and severe symptoms, 20-35). Uroflowmetry and postvoid residual (PVR) urine volume measurement were performed in all the patients. Uroflowmetry and PVR data were included in the analysis only if the participant excreted more than $150 \mathrm{~mL}$ of urine. TRUS of the prostate was performed to estimate total prostate volume (TPV) and transition zone volume (TZV), and the presence of intravesical prostatic protrusion (IPP). This was followed by power Doppler ultrasonography (PDUS) to identify the urethral artery, and left and right capsular arteries of the prostate and to measure their respective RIs (Fig. 1). We determined IPP by measuring the vertical distance from the wall of the bladder neck to the top of the prostate at the site of the maximal protrusion into the bladder on transabdominal ultrasonographic longitudinal images when the urine volume in the bladder was $200 \mathrm{~mL}$ or less. The patients were also categorized with respect to TPV ( $<30$ and $\geq 30 \mathrm{~mL}$ ). TRUS and PDUS were performed by one urologist by using a Medison Solidus
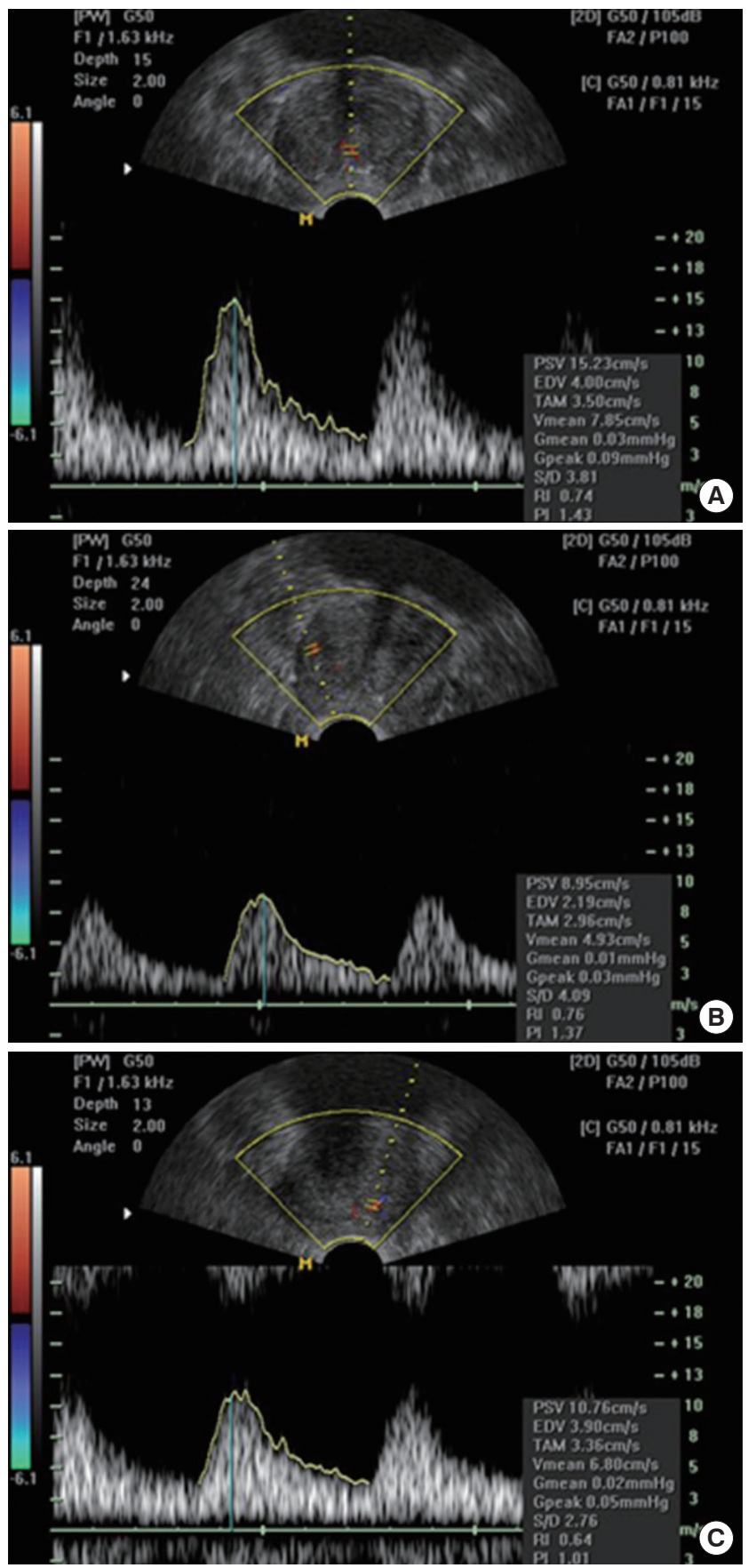

Fig. 1. Power Doppler ultrasonography of patient with benign prostatic hyperplasia and measurement of resistive index. (A) Urethral artery, (B) right capsular artery, and (C) left capsular artery.

Ex SA-8000 unit (Samsung Madison, Seoul, Korea).

The baseline parameters analyzed in the 110 study subjects included the RIs of the urethral artery, and left and right capsular arteries; IPSS (0-7, 8-19, and 20-35), quality-of-life score, 
maximal flow rate (Qmax; $<10 \mathrm{~mL} / \mathrm{sec}$ and $\geq 10 \mathrm{~mL} / \mathrm{sec}$ ), TPV $(<30$ and $\geq 30 \mathrm{~mL}), \mathrm{TZV}$, transition zone index $(\mathrm{TZI}=\mathrm{TZV} /$ $\mathrm{TPV}$ ), and the presence of IPP.

Statistical analyses were used to determine the RIs of the capsular and urethral arteries, and to investigate their relationships with the $\mathrm{BPH}$ parameters. Continuous variables were compared between the 2 groups by using the independent Student $t$-test. The relationships between the 2 parameters were analyzed by using Pearson correlation coefficients. All P-values were twosided, and P-values $<0.05$ were considered significant. The analysis was conducted by using IBM SPSS Statistics ver. 19.0 (IBM Co., Armonk, NY, USA).

\section{RESULTS}

The baseline characteristics of the 110 study subjects are summarized in Table 1. The mean patient age was $69.3 \pm 10.2$ years. The mean RI of the urethral artery was $0.62 \pm 0.08$, and the mean RIs of the left and right capsular arteries were $0.66 \pm 0.08$ and $0.65 \pm 0.09$, respectively. The mean IPSS was $17.7 \pm 9.6$;

Table 1. Baseline clinical characteristics

\begin{tabular}{lc}
\hline Characteristic & Mean \pm SD \\
\hline Age (yr) & $69.3 \pm 10.2$ \\
Resistive index & \\
Urethral artery & $0.62 \pm 0.08$ \\
Right capsular artery & $0.65 \pm 0.09$ \\
Left capsular artery & $0.66 \pm 0.08$ \\
International Prostate Symptom Score & $17.7 \pm 9.6$ \\
Prostate specific antigen $(\mathrm{ng} / \mathrm{mL})$ & $1.9 \pm 2.3$ \\
Maximal urinary flow rate $(\mathrm{mL} / \mathrm{sec})$ & $11.0 \pm 6.6$ \\
Prostate volume (mL) & $32.1 \pm 13.5$ \\
Transition zone volume $(\mathrm{mL})$ & $13.0 \pm 6.6$ \\
Transition zone index & $0.39 \pm 0.19$ \\
\hline
\end{tabular}

$\mathrm{SD}$, standard deviation.

Table 2. Relationship between IPSS and RI

\begin{tabular}{lccc}
\hline \multirow{2}{*}{ RI } & \multicolumn{2}{c}{ IPSS } & P-value \\
\cline { 2 - 3 } & $8-19(\mathrm{n}=65)$ & $20-35(\mathrm{n}=45)$ & \\
\hline Urethral artery & $0.62 \pm 0.08$ & $0.62 \pm 0.08$ & 0.75 \\
Right capsular artery & $0.64 \pm 0.09$ & $0.66 \pm 0.08$ & 0.16 \\
Left capsular artery & $0.65 \pm 0.09$ & $0.65 \pm 0.09$ & 0.89
\end{tabular}

Values are presented as mean \pm standard deviation.

IPSS, International Prostate Symptom Score; RI, resistive index. mean PSA, $1.9 \pm 2.3 \mathrm{ng} / \mathrm{mL}$; mean Qmax, $11.0 \pm 6.6 \mathrm{~mL} / \mathrm{sec}$; and mean TPV, $32.1 \pm 13.5 \mathrm{~mL}$.

The patients were divided according to IPSS as follows: mild symptom (0-7), moderate symptom (8-19), and severe symptom (20-35) groups. None of the patients had mild symptoms. In the moderate symptom group, the mean RIs of the urethral, right capsular, and left capsular arteries were $0.62 \pm 0.08$, $0.64 \pm 0.09$, and $0.65 \pm 0.09$. In the severe symptom group, the mean RIs were $0.62 \pm 0.08,0.66 \pm 0.08$, and $0.65 \pm 0.09$, respectively. No significant differences were observed between the IPSS and RIs (Table 2). The patients were also divided into 2 groups according to Qmax ( $<10 \mathrm{~mL} / \mathrm{sec}$ vs. $\geq 10 \mathrm{~mL} / \mathrm{sec}$ ). The mean RIs of the urethral, right capsular, and left capsular arteries were $0.65 \pm 0.07,0.64 \pm 0.11$, and $0.67 \pm 0.08$ for a Qmax of $<10 \mathrm{~mL} / \mathrm{sec}$, and $0.63 \pm 0.08,0.65 \pm 0.09$, and $0.64 \pm 0.08$ for a Qmax of $\geq 10 \mathrm{~mL} / \mathrm{sec}$, respectively. No significant differences were found between the Qmax and RIs (Table 3). The patients were further divided into 2 groups according to TPV ( $<30$ and $\geq 30 \mathrm{~mL}$ ). The mean RIs of the urethral, right capsular, and left capsular arteries were $0.60 \pm 0.08$ and $0.64 \pm 0.08,0.62 \pm 0.08$ and $0.65 \pm 0.08$, and $0.63 \pm 0.09$ and $0.67 \pm 0.09$, respectively, with significant differences between the 2 groups (Table 4 ).

For those with or without IPP, the mean RIs of the urethral, right capsular, and left capsular arteries were $0.69 \pm 0.05$ and $0.61 \pm 0.09(\mathrm{P}=0.004), 0.72 \pm 0.09$ and $0.65 \pm 0.09(\mathrm{P}=0.012)$, and $0.70 \pm 0.09$ and $0.63 \pm 0.07(\mathrm{P}=0.015)$, respectively, with

Table 3. Relationship between Qmax and RI

\begin{tabular}{lccc}
\hline \multirow{2}{*}{ RI } & \multicolumn{2}{c}{$\mathrm{Qmax}(\mathrm{mL} / \mathrm{sec})$} & \\
\cline { 2 - 3 } & $<10(\mathrm{n}=68)$ & $\geq 10(\mathrm{n}=42)$ & \\
\hline Urethral artery & $0.65 \pm 0.07$ & $0.63 \pm 0.08$ & 0.883 \\
Right capsular artery & $0.64 \pm 0.11$ & $0.65 \pm 0.09$ & 0.599 \\
Left capsular artery & $0.67 \pm 0.08$ & $0.64 \pm 0.08$ & 0.792 \\
\hline
\end{tabular}

Values are presented as mean \pm standard deviation. Qmax, maximal urinary flow rate; RI, resistive index.

Table 4. Relationship between prostate volume and RI

\begin{tabular}{lccc}
\hline \multirow{2}{*}{ RI } & \multicolumn{2}{c}{ Prostate volume $(\mathrm{mL})$} & \\
\cline { 2 - 3 } & $<30(\mathrm{n}=57)$ & $\geq 30(\mathrm{n}=53)$ & P-value \\
\hline Urethral artery & $0.60 \pm 0.08$ & $0.64 \pm 0.08$ & 0.010 \\
Right capsular artery & $0.62 \pm 0.08$ & $0.65 \pm 0.08$ & 0.045 \\
Left capsular artery & $0.63 \pm 0.09$ & $0.67 \pm 0.09$ & 0.030
\end{tabular}

Values are presented as mean \pm standard deviation.

RI, resistive index. 
Table 5. Relationship between IPP and RI

\begin{tabular}{lccc}
\hline RI & $\mathrm{IPP}(+)(\mathrm{n}=38)$ & $\mathrm{IPP}(-)(\mathrm{n}=72)$ & P-value \\
\hline Urethral artery & $0.69 \pm 0.05$ & $0.61 \pm 0.09$ & 0.004 \\
Right capsular artery & $0.72 \pm 0.09$ & $0.65 \pm 0.09$ & 0.012 \\
Left capsular artery & $0.70 \pm 0.09$ & $0.63 \pm 0.07$ & 0.015 \\
\hline
\end{tabular}

Values are presented as mean \pm standard deviation. IPP, intraprostatic protrusion; RI, resistive index.

significant differences between the 2 groups (Table 5).

No correlation was found between the RIs of the urethral artery and transition zone (TZ), and between the RIs of both capsular arteries and TZ. Similarly, no correlation was found between the RI of the urethral artery and TZI, and between the RI of both capsular arteries and TZI (Table 6).

\section{DISCUSSION}

TRUS is an optional study in BPH patients with complaints of LUTS and is commonly used to measure TPV and TZV. Advances in power Doppler imaging (PDI) have greatly enhanced abilities to detect and analyze Doppler signals of organ blood flow [13].

PDI, the next-generation color Doppler imaging, is based on the detection of signal amplitudes, which reflect red blood cell densities, independent blood flow rates, and flow directions [16]. The availability of power and color Doppler and transrectal probes improved the diagnosis of $\mathrm{BPH}$ by providing a means of assessing intraprostatic vascularity [14]. Currently, the use of transrectal probes in power and color Doppler ultrasonographies allows detailed assessment of intraprostatic vascularity [15].

Nelson and Pretorius [17] showed that RIs obtained by using Doppler imaging correlates with vascular resistance because velocity is related to blood flow and pressure. Accordingly, RI might be the most relevant index for analyzing small vessels in the prostate. With the advent of PDI, RI measurements in patients with LUTS offer a promising means of diagnosing $\mathrm{BPH}$ [18].

The urethral arteries are components of the inferior vesical arterial system and form an angle of $90^{\circ}$ to enfold into the prostate through the bladder neck. On the other hand, the capsular arteries originate from the prostatic arteries as they course along the anterolateral surface of the gland [12]. In general, the RI of a normal prostate might become the same anywhere, but
Table 6. Relationship between TZ and TZI

\begin{tabular}{lcccccc}
\hline \multirow{2}{*}{ RI } & \multicolumn{2}{c}{ TZ } & & \multicolumn{2}{c}{ TZI } \\
\cline { 2 - 3 } \cline { 5 - 6 } & $r$ & P-value & & $r$ & P-value \\
\hline Urethral artery & 0.319 & 0.155 & & 0.307 & 0.152 \\
Right capsular artery & 0.267 & 0.219 & & 0.238 & 0.126 \\
Left capsular artery & 0.171 & 0.289 & & 0.249 & 0.142 \\
\hline
\end{tabular}

TZ, transition zone; TZI, transition zone index; RI, resistive index.

when prostatic enlargement is advanced, blood flow between the peripheral and transitional zones are reduced by compression, and this causes a marked increase in the RIs of the capsular arteries [16]. The mechanisms responsible for the increases in the RIs of the capsular arteries in patients with elevated TZI and presumed circle area ratios (PCAR) have not been clarified. Some reports suggest that the mechanical obstruction in $\mathrm{BPH}$ is closely related to the $\mathrm{TZ}$, especially to compression of the peripheral zone by $\mathrm{TZ}$ enlargement [10].

In a previous study, increases in the RIs of the capsular arteries correlated with increases in TZI and PCAR in BPH, and IPSS and peak flow rate were inversely correlated capsular artery RIs, but no correlation was found between the RIs of the urethral artery and any of the prostatic parameters [16]. However, in the present study, RI and TPV were positively associated, and the presence of IPP was related to RI, but Qmax, TZ, and TZI were not.

Hayami et al. [19] assessed the predictive value of PDI for histological components of $\mathrm{BPH}$ and demonstrated that RI most reliably predicted the ratio of glandular lumen to stromal elements. These values are considered useful for predicting therapeutic response.

In a previous study, RI of the prostate vasculature differentiated patients with a normal prostate from those with $\mathrm{BPH}$ and was proposed to be a useful new hemodynamic parameter [20]. Kojima et al. [9] showed that RI significant correlated with urodynamic parameters and IPSS. The RIs of the capsular arteries, and urethral artery that were measured on Doppler imaging were found to correlate with vascular resistance, presumably because blood velocity is related to both blood flow and pressure. This suggests that RI might be the most relevant index for analyzing small vessels in the prostate, as has been previously suggested [17], as BPH increases vascular resistance and prostatic RI increases due to prostate enlargement [14].

Increased prostatic RI in patients with LUTS and BPH may be due to increased intraprostatic pressure caused by prostatic 
hypertrophy, which concurs with previous findings that prostatic RI in patients is correlated positively with TPV and IPSS, and negatively with Qmax [21]. Furthermore, the RIs of prostate capsular arteries have been suggested to enable the diagnosis of bladder outlet obstruction in patients with BPH [22].

The present study shows that prostatic RI was statistically significant related with TPV and IPP, but not with the other variables examined. This may have been because blood flow was slower when TPV was larger and the presence of IPP increased arterial vascular resistance. Moreover, the RIs obtained in our study were lower than those obtained in previous studies because of the relatively small size of the prostates of our participants. Therefore, these results are important because they demonstrate the benefit of adding prostatic RI to conventional diagnostic and follow-up assessments of IPSS and Qmax in patients with LUTS and BPH.

Bulut et al. [13] reported a posttreatment decrease in prostatic RI in LUTS/BPH, which may be associated with a decrease in intraprostatic pressure. They found that prostatic RIs correlated positively with TPV and IPSS, and negatively with Qmax, which is similar to that reported in previous studies [18]. However, the reason for the increase in RI in BPH has not been established, but it may be because the growing hypertrophic prostate pushes the capsule outward and thereby increases intraprostatic pressure and RIs [23].

This study has some limitations. Our results are based on a relatively small sample size. In addition, multivariate analysis was not performed. Nevertheless, our study has a prospective design, and we were able to show that RI is associated with $\mathrm{BPH}$. Further studies in a large number of patients would clarify the clinical relationship of RIs to the diagnosis of BPH with LUTS. Moreover, the aspect of change in RIs after management of BPH such as with medication or surgery should be investigated.

In conclusion, RI obtained by using PDUS correlated with the presence of IPP and prostate size. The RI of prostate blood flow can be used as a noninvasive diagnostic tool for BPH with LUTS.

\section{REFERENCES}

1. AUA Practice Guidelines Committee. AUA guideline on management of benign prostatic hyperplasia (2003). Chapter 1: diagnosis and treatment recommendations. J Urol 2003;170(2 Pt 1):530-47.

2. Park HJ, Won JE, Sorsaburu S, Rivera PD, Lee SW. Urinary tract symptoms (LUTS) secondary to benign prostatic hyperplasia (BPH) and LUTS/BPH with erectile dysfunction in Asian men: a systematic review focusing on tadalafil. World J Mens Health 2013;31:193-207.

3. Park SK, Sakoda LC, Kang D, Chokkalingam AP, Lee E, Shin HR, et al. Rising prostate cancer rates in South Korea. Prostate 2006;66:128591.

4. Son H, Park J, Song SH, Kang JY, Hong SK, Lee HM, et al. Rapid increase of health care utilization and cost due to benign prostatic hyperplasia in Korean men: retrospective population-based analysis using the Health Insurance Review and Assessment service data. J Korean Med Sci 2015;30:180-5.

5. Park J, Lee YJ, Lee JW, Yoo TK, Chung JI, Yun SJ, et al. Comparative analysis of benign prostatic hyperplasia management by urologists and nonurologists: a Korean nationwide health insurance database study. Korean J Urol 2015;56:233-9.

6. Parsons JK. Benign prostatic hyperplasia and male lower urinary tract symptoms: epidemiology and risk factors. Curr Bladder Dysfunct Rep 2010;5:212-8.

7. Robert G, Descazeaud A, Azzouzi R, Saussine C, Haillot O, Dumonceau $\mathrm{O}$, et al. Impact of lower urinary tract symptoms on discomfort in men aged between 50 and 80 years. Urol Int 2010;84:424-9.

8. Kojima M, Naya Y, Inoue W, Ukimura O, Watanabe M, Saitoh M, et al. The American Urological Association symptom index for benign prostatic hyperplasia as a function of age, volume and ultrasonic appearance of the prostate. J Urol 1997;157:2160-5.

9. Kojima M, Ochiai A, Naya Y, Ukimura O, Watanabe M, Watanabe H. Correlation of presumed circle area ratio with infravesical obstruction in men with lower urinary tract symptoms. Urology 1997; 50:548-55.

10. Kurita Y, Masuda H, Terada H, Suzuki K, Fujita K. Transition zone index as a risk factor for acute urinary retention in benign prostatic hyperplasia. Urology 1998;51:595-600.

11. Rifkin MD, Sudakoff GS, Alexander AA. Prostate: techniques, results, and potential applications of color Doppler US scanning. Radiology 1993;186:509-13.

12. Neumaier CE, Martinoli C, Derchi LE, Silvestri E, Rosenberg I. Normal prostate gland: examination with color Doppler US. Radiology 1995;196:453-7.

13. Bulut S, Ozden C, Aktas BK, Deren T, Tagci S, Gokkaya CS, et al. Effects of medical therapy or surgery on prostatic and bladder resistive indices in patients with benign prostatic hyperplasia. Urol Int 2015;94:181-6.

14. Shinbo H, Kurita Y. Application of ultrasonography and the resistive index for evaluating bladder outlet obstruction in patients with 
benign prostatic hyperplasia. Curr Urol Rep 2011;12:255-60.

15. Tsuru N, Kurita Y, Masuda H, Suzuki K, Fujita K. Role of Doppler ultrasound and resistive index in benign prostatic hypertrophy. Int J Urol 2002;9:427-30.

16. Amiel GE, Slawin KM. Newer modalities of ultrasound imaging and treatment of the prostate. Urol Clin North Am 2006;33:329-37.

17. Nelson TR, Pretorius DH. The Doppler signal: where does it come from and what does it mean? AJR Am J Roentgenol 1988;151:43947.

18. Ozdemir H, Onur R, Bozgeyik Z, Orhan I, Ogras MS, Ogur E. Measuring resistance index in patients with $\mathrm{BPH}$ and lower urinary tract symptoms. J Clin Ultrasound 2005;33:176-80.

19. Hayami S, Ushiyama T, Kurita Y, Kageyama S, Suzuki K, Fujita K. The value of power Doppler imaging to predict the histologic components of benign prostatic hyperplasia. Prostate 2002;53:168-74.
20. Kojima M, Watanabe H, Watanabe M, Okihara K, Naya Y, Ukimura O. Preliminary results of power Doppler imaging in benign prostatic hyperplasia. Ultrasound Med Biol 1997;23:1305-9.

21. Ozden C, Gunay I, Deren T, Bulut S, Ozdal OL, Koparal S, et al. Effect of transurethral resection of prostate on prostatic resistive index. Urol Int 2010;84:191-3.

22. Zhang X, Li G, Wei X, Mo X, Hu L, Zha Y, et al. Resistive index of prostate capsular arteries: a newly identified parameter to diagnose and assess bladder outlet obstruction in patients with benign prostatic hyperplasia. J Urol 2012;188:881-7.

23. Berger AP, Horninger W, Bektic J, Pelzer A, Spranger R, Bartsch G, et al. Vascular resistance in the prostate evaluated by colour Doppler ultrasonography: is benign prostatic hyperplasia a vascular disease? BJU Int 2006;98:587-90. 